\title{
Impact of the estimation equation for GFR on population-based prevalence estimates of kidney dysfunction
}

\author{
Pietro Trocchi ${ }^{* *}$, Matthias Girndt ${ }^{2}$, Christa Scheidt-Nave 3 , Silke Markau² and Andreas Stang ${ }^{1,4}$
}

\begin{abstract}
Background: Estimating equations are recommended by clinical guidelines as the preferred method for assessment of glomerular filtration rate (GFR). The aim of the study was to compare population-based prevalence estimates of decreased kidney function in Germany defined by an estimated GFR (eGFR) $<60 \mathrm{ml} / \mathrm{min} / 1.73 \mathrm{~m}^{2}$ using different equations.

Methods: The study included 7001 participants of the German Health Interview and Examination Survey for Adults 2008-2011 (DEGS1) for whom GFR was estimated using the Modification of Diet in Renal Disease study equation (MDRD), the revised Lund-Malmö equation (LM), the Full Age Spectrum creatinine equation (FAScre), the Chronic Kidney Disease Epidemiology Collaboration equations with creatinine and cystatin C (CKD-EPIcrecys), with creatinine (CKD-EPIcre) and with cystatin C (CKD-EPIcys). Bland-Altman plots were used to evaluate the agreement between the equations.

Results: Prevalence estimates of decreased kidney function were: 2.1\% (CKD-EPIcys), 2.3\% (CKD-EPIcrecys), 3.8\% (CKD-EPIcre), 5.0\% (MDRD), 6.0\% (LM) and 6.9\% (FASCre). The systematic differences between the equations were smaller by comparing either equations that include serum cystatin $C$ or equations that include serum creatinine alone and increased considerably by increasing eGFR.

Conclusions: Prevalence estimates of decreased kidney function vary considerably according to the equation used for estimating GFR. Equations that include serum cystatin C provide lower prevalence estimates if compared with equations based on serum creatinine alone. However, the analysis of the agreement between the equations according to eGFR provides evidence that the equations may be used interchangeably among persons with pronounced decreased kidney function. The study illustrates the implications of the choice of the estimating equation in an epidemiological setting.
\end{abstract}

Keywords: Epidemiology, Prevalence, Renal dysfunction, eGFR equation

\section{Background}

Chronic Kidney Disease (CKD) is defined by morphological and functional damage to the kidney $[1,2]$. Clinical assessment of kidney function is central to the routine clinical practice $[3,4]$ and glomerular filtration rate (GFR) is the best overall index-indicator of

\footnotetext{
* Correspondence: pietro.trocchi@uk-essen.de

${ }^{1}$ Center of Clinical Epidemiology, c/o Institute of Medical Informatics, Biometry and Epidemiology (IMIBE), University Hospital Essen, Hufelandstr. 55, 45147 Essen, Germany

Full list of author information is available at the end of the article
}

excretory kidney function in health and disease $[3,5]$. In the epidemiological setting, decreased kidney function may be defined by a GFR $<60 \mathrm{ml} / \mathrm{min} / 1.73 \mathrm{~m}^{2}$ [1]. As directly measuring GFR is often cumbersome in routine clinical practice, researchers have developed and validated several GFR estimating equations that include demographic and clinical variables as surrogates for muscle mass and unmeasured factors that affect serum creatinine level, such as age, sex and race. Some of these equations are meanwhile recommended by clinical guidelines as the preferred method for assessment of GFR in the routine clinical care [1]. 
Decreased kidney function has been shown to be an independent marker for major adverse outcomes of CKD, including progression to end-stage kidney failure and premature death caused by cardiovascular disease [6-8]. Patients with decreased kidney function require considerable medical attention to prevent deterioration and the development of complications. If kidney disease progresses to end-stage kidney disease, renal replacement therapy is an enormously resource consuming condition. Given its high impact on patients' quality of life and medical resources and rising prevalence estimates reported from many countries [9], CKD is increasingly recognized a major public health problem and the knowledge of its prevalence is of great importance from both a medical and the economical standpoint. Several studies evaluated performance and limitations of different estimating equations for GFR against a gold standard of kidney function testing in a clinical setting $[4,10-14]$. Although it is widely known to nephrologist that the eGFR equations perform differently in relation to patient characteristics, the behavior of the equations in unselected large population-based samples has only been investigated in detail in the NHANES study to our knowledge. Therefore, a detailed assessment of the behavior of the equations in a European, predominantly Caucasian population of 7000 participants is important for researchers who want to provide population-based prevalence estimates of kidney dysfunction. To date, literature about the prevalence of kidney function in Germany is scarce. Recently, we published populationbased estimates of prevalence of kidney damage in Germany based on measures of albuminuria and the use of an established equation for GFR estimation [15]. Furthermore, the Study of Health in Pomerania (SHIP-1) and the Cooperative Health Research in the Region of Augsburg (KORA F4) reported results about prevalence of decreased kidney function in Northeast and Southern Germany respectively [16]. Finally, the Berlin Initiative Study assessed kidney function in Berlin in a cross sectional analysis of people aged 70 years and older [17]. The present study compares different population-based prevalence estimates of decreased kidney function among adults in Germany using six different GFR estimating equations.

\section{Methods}

\section{Study population and design}

The German Health Interview and Examination Survey for Adults ("Studie zur Gesundheit Erwachsener in Deutschland", DEGS) is part of the health monitoring system at the Robert Koch-Institute (RKI). The concept and design of DEGS are described in detail elsewhere [18-20]. The first wave (DEGS1) was conducted from 2008 to 2011 and included interviews, examinations and tests. The DEGS1 study has a mixed design, which enables both cross-sectional and longitudinal analyses. For this purpose, a random sample from local population registries was drawn to supplement former participants from the German National Health Interview and Examination Survey 1998 (GNHIES98). To evaluate kidney function, blood samples were taken from all participants and serum creatinine concentration (Architect, Abbott Diagnostics, Wiesbaden; IDMS traceable creatinine Assay, kinetic Jaffe's method) and serum cystatin $C$ concentration (N Latex Cystatin C assay, Prospec, Siemens Healthcare, Eschborn) were measured. Participants with diabetes mellitus were identified according to self-reported medical history and verified current use of anti-diabetic drugs [21]. Participants with gestational diabetes were not included among those with diabetes mellitus. Arterial hypertension was assumed if the participant reported current treatment with antihypertensive medications or elevated blood pressure ( $\geq 140 \mathrm{mmHg}$ systolic or $\geq 90 \mathrm{mmHg}$ diastolic) was measured in the survey [22].

The analyses presented here refer to the sample of 7001 participants of the DEGS1 aged 18-79 years for whom estimated GFR (eGFR) was calculated using six GFR estimating equations (creatinine was measured in $\mathrm{mg} / \mathrm{dl}$, cystatin $\mathrm{C}$ in $\mathrm{mg} / \mathrm{l}$ ): the isotope dilution mass spectrometry traceable Modification of Diet in Renal Disease study equation (MDRD) [23], the revised LundMalmö equation (LM) [12], the Full Age Spectrum creatinine equation (FAScre) [24], the Chronic Kidney Disease Epidemiology Collaboration creatinine equation (CKD-EPIcre), the Chronic Kidney Disease Epidemiology Collaboration cystatin C equation (CKD-EPIcys) and the Chronic Kidney Disease Epidemiology Collaboration creatinine and cystatin $\mathrm{C}$ equation (CKD-EPIcrecys) [4]. Equations are detailed in Fig. 1. Persons with missing data on eGFR were excluded $(N=114)$.

\section{Statistical analysis}

Participants were classified into four GFR categories based on the estimated GFR values (expressed in $\mathrm{ml} /$ $\left.\mathrm{min} / 1.73 \mathrm{~m}^{2}\right)$ as follows: G1 $(\geq 90), \mathrm{G} 2(60<90), \mathrm{G} 3 \mathrm{a}(45$ $<60)$ and G3b-G5 $(<45)$. We estimated the prevalence and corresponding $95 \%$ confidence intervals $(95 \% \mathrm{CI}$ ) of a decreased kidney function, as defined by an eGFR $<60 \mathrm{ml} / \mathrm{min} / 1.73 \mathrm{~m}^{2}$, and the prevalence of each GFR category. Furthermore, we calculated the population based estimate of the number of persons with decreased kidney function in Germany using the population census figures of the Federal Republic of Germany in 2011. Population data were provided by the Federal Bureau of Statistic.

We evaluated the agreement between the six equations used for estimating GFR according to the approach proposed by Bland and Altman [25]. Bland-Altman plots 


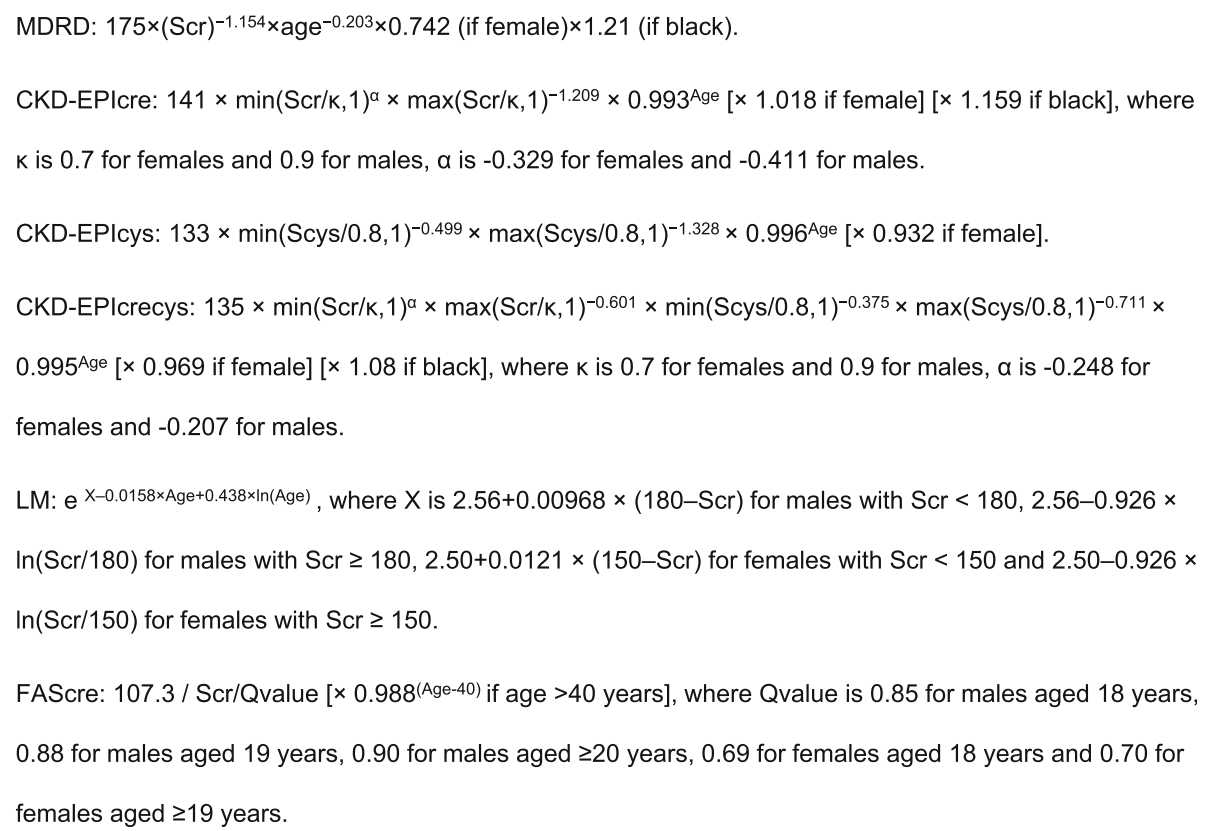

Fig. 1 Equations used to estimate GFR. MDRD: Modification of Diet in Renal Disease study equation; CKD-EPIcre: Chronic Kidney Disease Epidemiology Collaboration creatinine equation; CKD-EPIcys: Chronic Kidney Disease Epidemiology Collaboration cystatin C equation; CKD-EPIcrecys: Chronic Kidney Disease Epidemiology Collaboration creatinine and cystatin C equation; LM: Lund-Malmö equation; FAScre: Full Age Spectrum creatinine equation; Scr: serum creatinine; Scys: serum cystatin C; min: minimum; max: maximum

display for each person the difference between two measurements against their mean. To compare the equations with each other, the absolute mean difference (md) between eGFR was used as a measure of the magnitude of the systematic difference (bias) between the equations. In addition, as a measure of relative change of eGFR, we calculated the mean percent difference between the measurements. The limits of the agreement between the equations were defined as $\mathrm{md} \pm 1.96$ standard deviation of the differences (SD) and were used as a measure of the variability of the bias. These values represented the range within which $95 \%$ of the differences were included (95\% CI agreement). Furthermore, for each of the ten pairwise comparisons, the analysis of the md was stratified by the GFR category based on the mean value of eGFR (G1: $\geq 90$, G2: $60<90$, G3a: $45<60$, G3b-G5: <45).

Prevalence estimates were weighted by a factor that corrects sample deviations from population structure (as of 31 Dec. 2010) with regard to age, sex, region and nationality, type of community and education. When calculating the weighting factor for previous participants of GNHIES98, the probability of repeated participation, based on a multivariable logistic model, was taken into account. A non-response analysis and a comparison of selected indicators with data from official statistics indicate a high level of sample representativeness for the resident population of Germany aged 18-79 years [18]. In addition, the observed number of subjects end stage renal disease in our sample $(n=1)$ was similar to the expected number of subjects with end stage renal disease $(n=7)$ as derived from a recent report [26]. To take into account both the weighting and the correlation of the participants within a community, confidence intervals were determined using the survey procedures in $\mathrm{SAS}^{\circ}$ (SAS Inc., Cary, NC, USA), Version 9.4.

\section{Results}

Table 1 shows details about the main characteristics of the 7001 participants of the DEGS1 study from whom the GFR was estimated using different eqs. (3364 men and 3637 women). The median age was 46.9 years (men: 46.4, women: 47.5). The prevalence estimates of persons with medical history of arterial hypertension and diabetes mellitus were $31.5 \%$ and $6.6 \%$ respectively. Table 2 displays the estimated prevalence of participants with decreased kidney function, defined as eGFR $<60 \mathrm{ml} /$ $\min / 1.73 \mathrm{~m}^{2}$, stratified by sex and age group. Overall, the prevalence of participants aged 18-79 years with decreased kidney function differed considerably depending on the equation used and was as follows: $2.1 \%$ (CKDEPIcys), 2.3\% (CKD-EPIcrecys), 3.8\% (CKD-EPIcre), 5.0\% (MDRD), 6.0\% (LM) and 6.9\% (FAScre). The prevalence ranged from $1.6 \%$ to $5.6 \%$ among men and from $2.6 \%$ to $8.2 \%$ among women using the CKD-EPIcys equation and the FAScre equation respectively. Whatever equation was used, the estimated prevalence was 
Table 1 Characteristics of 7001 adults aged 18-79 in Germany 2008-2011 (DEGS1)

\begin{tabular}{|c|c|c|c|c|c|c|}
\hline \multirow{2}{*}{$\begin{array}{l}\text { Characteristic } \\
\text { Sex: N, \% }\end{array}$} & \multicolumn{2}{|c|}{ Overall } & \multicolumn{2}{|l|}{ Men } & \multicolumn{2}{|c|}{ Women } \\
\hline & 7001 & 100 & 3364 & 49.9 & 3637 & 50.1 \\
\hline Age (Years): median (P10, P90) & \multicolumn{2}{|c|}{$46.9(23.6,70.4)$} & \multicolumn{2}{|c|}{$46.4(23.3,69.8)$} & \multicolumn{2}{|c|}{$47.5(23.9,70.9)$} \\
\hline BMI (Kg/m²): median (P10, P90) & \multicolumn{2}{|c|}{$26.2(21.0,33.4)$} & \multicolumn{2}{|c|}{$26.7(22.1,32.9)$} & \multicolumn{2}{|c|}{$25.4(20.4,33.8)$} \\
\hline Serum creatinine $(\mathrm{mg} / \mathrm{dl})$ : median (P10, P90) & \multicolumn{2}{|c|}{$0.82(0.67,1.06)$} & \multicolumn{2}{|c|}{$0.92(0.76,1.13)$} & \multicolumn{2}{|c|}{$0.75(0.63,0.90)$} \\
\hline Serum cystatin C (mg/l): median (P10, P90) & \multicolumn{2}{|c|}{$0.70(0.57,0.90)$} & \multicolumn{2}{|c|}{$0.73(0.61,0.90)$} & \multicolumn{2}{|c|}{$0.67(0.55,0.89)$} \\
\hline \multicolumn{7}{|l|}{ Medical history } \\
\hline Hypertension: N, \% & 2585 & 31.5 & 1349 & 33.4 & 1236 & 29.7 \\
\hline Diabetes mellitus: N, \% & 539 & 6.6 & 305 & 7.0 & 234 & 6.2 \\
\hline
\end{tabular}

P10 10th percentile, $P 90$ 90th percentile, $B M I$ body mass index

higher for women than for men and increased with age in both sexes: among participants aged $<60$ years the prevalence varied from $0.2 \%$ to $1.2 \%$ and increased among participants aged $70<80$ years up to $11.4 \%$ using CKD-EPIcys and up to $38.4 \%$ using FAScre. The mean eGFR varied from 83.7 using LM to 111.4 using CKDEPIcys.

Based on the age specific prevalence estimates and the German population in 2011, the estimated number of persons aged 18-79 years with decreased kidney function in Germany varied from $1.41 \mathrm{~m}$ using the CKDEPIcys equation to $4.58 \mathrm{~m}$ using the FAScre equation. Assuming that the prevalence of persons aged $\geq 80$ with decreased kidney function equals that of the study participants aged $75<80$ years, the total number of persons with decreased kidney function ranged from $2.15 \mathrm{~m}$ using the CKD-EPIcys equation to $6.78 \mathrm{~m}$ using the FAScre equation (Table 3).

Table 2 eGFR and estimated prevalence of decreased kidney function (eGFR $<60 \mathrm{ml} / \mathrm{min} / 1.73 \mathrm{~m}^{2}$ ) among 7001 adults aged $18-79$ in Germany 2008-2011 (DEGS1) according to the equation used

\begin{tabular}{|c|c|c|c|c|c|}
\hline \multirow{2}{*}{ 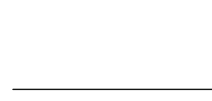 } & \multirow{2}{*}{ eGFR: Mean (SD, CV) } & \multicolumn{4}{|c|}{ Prevalence $(95 \% \mathrm{Cl})$} \\
\hline & & Overall & $<60$ years & $60<69$ years & $70<79$ years \\
\hline Overall & & $N=7001$ & $N=4538$ & $N=1376$ & $N=1087$ \\
\hline MDRD & $88.4(59.3,0.67)$ & $5.0(4.3-5.7)$ & $1.2(0.8-1.5)$ & $10.9(8.8-13.1)$ & $20.6(17.3-23.9)$ \\
\hline CKD-EPIcre & $95.2(40.9,0.43)$ & $3.8(3.3-4.4)$ & $0.4(0.2-0.6)$ & $7.8(5.9-9.6)$ & $19.2(16.1-22.4)$ \\
\hline CKD-EPIcys & $111.4(34.3,0.31)$ & $2.1(1.7-2.5)$ & $0.2(0.1-0.3)$ & $3.8(2.3-5.4)$ & $11.4(8.9-13.9)$ \\
\hline CKD-EPIcrecys & $105.1(34.5,0.33)$ & $2.3(1.9-2.7)$ & $0.2(0.1-0.3)$ & $3.8(2.2-5.3)$ & $12.8(10.4-15.3)$ \\
\hline LM & $83.7(25.3,0.43)$ & $6.0(5.3-6.7)$ & $0.5(0.3-0.8)$ & $11.7(9.5-13.8)$ & $31.4(27.6-35.2)$ \\
\hline FAScre & $91.7(54.8,0.60)$ & $6.9(6.1-7.6)$ & $0.5(0.3-0.7)$ & $11.9(9.9-14.0)$ & $38.4(34.2-42.5)$ \\
\hline Men & & $N=3364$ & $N=2150$ & $N=667$ & $N=547$ \\
\hline MDRD & $91.4(45.7,0.50)$ & $4.1(3.3-4.8)$ & $0.9(0.5-1.3)$ & $9.4(6.4-12.4)$ & $18.3(14.4-22.1)$ \\
\hline CKD-EPIcre & $96.4(32.2,0.34)$ & $3.6(2.9-4.4)$ & $0.5(.0 .2-0.8)$ & $7.7(4.9-10.4)$ & $19.0(14.9-23.1)$ \\
\hline CKD-EPIcys & $113.5(28.1,0.25)$ & $1.6(1.1-2.0)$ & $0.2(0.0-0.4)$ & $2.8(0.7-4.8)$ & $8.6(5.8-11.4)$ \\
\hline CKD-EPIcrecys & $106.8(27.6,0.26)$ & $1.7(1.3-2.2)$ & $0.3(0.1-0.5)$ & $2.3(0.3-4.3)$ & $10.5(7.6-13.4)$ \\
\hline LM & $84.0(28.1,0.33)$ & $5.6(4.8-6.5)$ & $0.5(0.3-0.8)$ & $12.3(9.1-15.5)$ & $30.5(25.6-35.5)$ \\
\hline FAScre & $94.2(43.8,0.46)$ & $5.6(4.7-6.4)$ & $0.4(0.1-0.6)$ & $11.3(8.2-14.4)$ & $32.1(27.0-37.1)$ \\
\hline Women & & $N=3637$ & $N=2388$ & $N=709$ & $N=540$ \\
\hline MDRD & $85.5(43.5,0.51)$ & $5.9(4.9-6.9)$ & $1.5(0.9-2.1)$ & $12.4(9.3-15.5)$ & $22.6(17.9-27.3)$ \\
\hline CKD-EPIcre & $94.0(32.6,0.35)$ & $4.0(3.2-4.8)$ & $0.3(0.1-0.6)$ & $7.8(5.3-10.4)$ & 19.4 (14.9-24.0) \\
\hline CKD-EPIcys & $109.3(29.6,0.27)$ & $2.6(2.0-3.3)$ & $0.1(0.0-0.2)$ & $4.9(2.4-7.4)$ & $13.8(9.7-17.8)$ \\
\hline CKD-EPIcrecys & $103.4(30.0,0.29)$ & $2.8(2.2-3.4)$ & $0.1(0.0-0.1)$ & $5.2(2.8-7.6)$ & $14.8(10.9-18.7)$ \\
\hline LM & $83.4(27.4,0.33)$ & $6.4(5.4-7.3)$ & $0.5(0.2-0.9)$ & $11.1(8.2-14.0)$ & $32.2(27.2-37.1)$ \\
\hline FAScre & $89.3(40.8,0.46)$ & $8.2(7.1-9.2)$ & $0.5(0.2-0.9)$ & $12.6(9.7-15.5)$ & $43.6(38.3-49.0)$ \\
\hline
\end{tabular}

SD standard deviation, CV coefficient of variation, Cl confidence interval, MDRD Modification of Diet in Renal Disease study equation, CKD-EPIcre Chronic Kidney Disease Epidemiology Collaboration creatinine equation, CKD-EPIcys Chronic Kidney Disease Epidemiology Collaboration cystatin C equation, CKD-EPIcrecys Chronic Kidney Disease Epidemiology Collaboration creatinine and cystatin C equation, LM Lund-Malmö equation, FAScre Full Age Spectrum creatinine equation 
Table 3 Estimated numbers (millions) of adults with decreased kidney function (eGFR $<60 \mathrm{ml} / \mathrm{min} / 1.73 \mathrm{~m}^{2}$ ) in Germany 2011 according to the equation used

\begin{tabular}{lllllll}
\hline Age (years) & MDRD & CKD-EPIcre & CKD-EPIcys & CKD-EPIcrecys & LM & FAScre \\
\hline$<50$ & 0.179 & 0.039 & 0.036 & 0.022 & 0.041 & 0.016 \\
$50<60$ & 0.373 & 0.162 & 0.037 & 0.058 & 0.214 & 0.199 \\
$60<70$ & 0.944 & 0.665 & 0.324 & 0.320 & 1.119 & 0.999 \\
$70<80$ & 1.772 & 1.672 & 0.745 & 0.737 & 2.773 \\
$\geq 80^{a}$ & 1.067 & 1.070 & 2.154 & 2.256 & 1.930 \\
Overall & 4.335 & 3.608 & & 5.958 & 2.349 \\
\hline
\end{tabular}

MDRD Modification of Diet in Renal Disease study equation, CKD-EPIcre Chronic Kidney Disease Epidemiology Collaboration creatinine equation, CKD-EPIcys Chronic Kidney Disease Epidemiology Collaboration cystatin C equation, CKD-EPIcrecys Chronic Kidney Disease Epidemiology Collaboration creatinine and cystatin $C$ equation, $L M$ Lund-Malmö equation, FAScre: Full Age Spectrum creatinine equation

${ }^{\mathrm{a}}$ By use of the estimated prevalence of study participants aged $70<80$ years

Figure 2 presents the prevalence estimates of the GFR categories according to the equation used. Using any CKD-EPI equations, the large majority of the participants were classified as G1. In particular, according to CKD-EPIcys, almost 9 out of 10 participants were classified in this category. Using MDRD or LM the highest prevalence was estimated for the participants with eGFR $60<90 \mathrm{ml} / \mathrm{min} / 1.73 \mathrm{~m}^{2}$ (category: G2). The prevalence of participants with eGFR $<45 \mathrm{ml} / \mathrm{min} / 1.73 \mathrm{~m}^{2}$ (category G3b-G5) varied from $0.7 \%$ using CKD-EPIcys to $1.4 \%$ using FAScre.
Table 4 shows that great absolute differences between eGFR were calculated comparing CKD-EPIcys with LM $(m d=27.6)$, CKD-EPIcys with MDRD $(m d=22.9)$ and CKD-EPIcrecys with LM $(\mathrm{md}=21.4)$. In contrast, small differences were calculated comparing FAScre with MDRD ( $m d=3.3$ ) and CKD-EPIcre ( $m d=3.5$ ), MDRD with LM ( $\mathrm{md}=4.7)$, CKD-EPIcys with CKD-EPIcrecys $(\mathrm{md}=6.2)$ and CKD-EPIcre with MDRD ( $\mathrm{md}=6.8$ ). Overall, the absolute md between eGFR were very small for small values of eGFR and increased considerably with increasing eGFR values. In particular, among

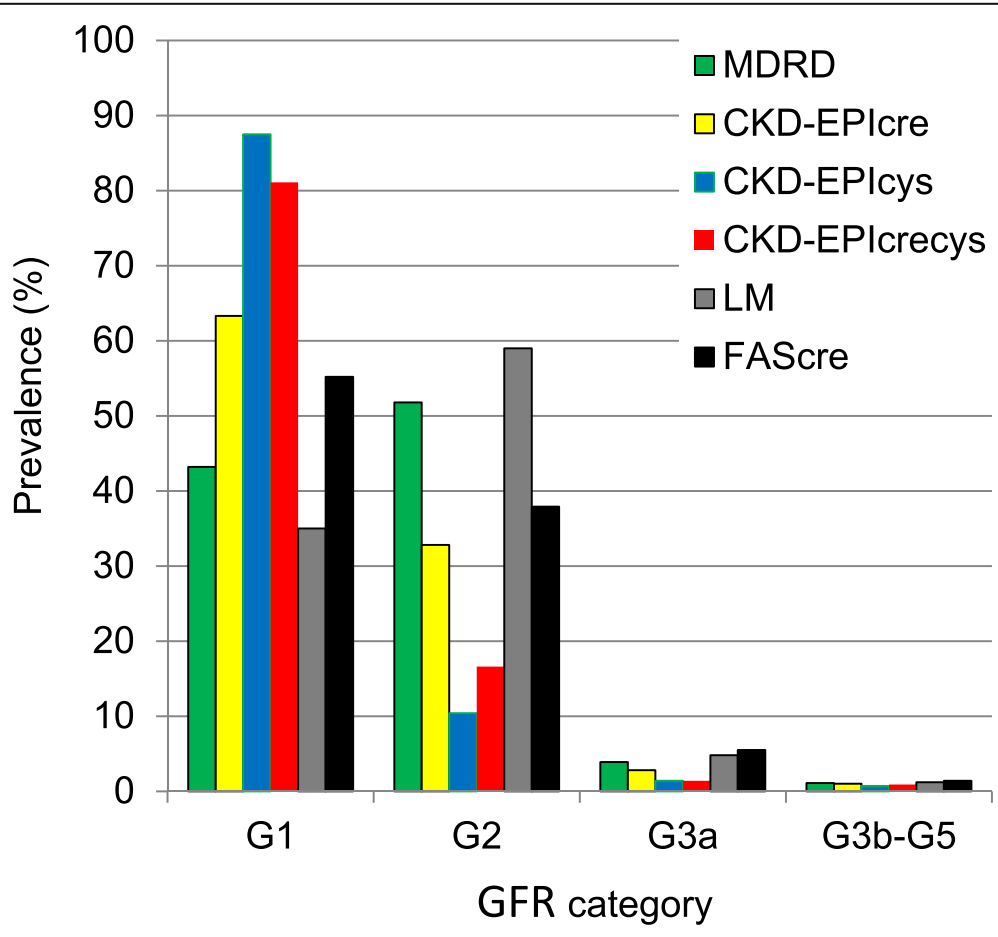

Fig. 2 Prevalence estimates of GFR categories among 7001 adults aged 18-79 in Germany 2008-2011 (DEGS1) according to the equation used. MDRD: Modification of Diet in Renal Disease study equation; CKD-EPIcre: Chronic Kidney Disease Epidemiology Collaboration creatinine equation; CKD-EPIcys: Chronic Kidney Disease Epidemiology Collaboration cystatin C equation; CKD-EPIcrecys: Chronic Kidney Disease Epidemiology Collaboration creatinine and cystatin C equation; LM: Lund-Malmö equation; FAScre: Full Age Spectrum creatinine equation 
Table 4 Absolute mean differences between GFR estimated by the different equations used among 7001 adults aged $18-79$ in Germany 2008-2011 (DEGS1) according to GFR category

\begin{tabular}{|c|c|c|c|c|c|}
\hline & Overall & GFR category & & & \\
\hline & & $\mathrm{G} 1$ & G2 & G3a & G3b-G5 \\
\hline MDRD vs. CKD-EPIcrecys & $16.7(15.9 \%)$ & $17.5(15.1 \%)$ & $16.1(18.3 \%)$ & $6.4(11.1 \%)$ & $2.2(6.0 \%)$ \\
\hline MDRD vs. CKD-EPIcre & $6.8(7.1 \%)$ & $7.1(6.6 \%)$ & $6.9(8.4 \%)$ & $1.7(3.1 \%)$ & $0.3(0.8 \%)$ \\
\hline MDRD vs. CKD-EPIcys & $22.9(20.6 \%)$ & $24.3(20.4 \%)$ & $20.0(22.2 \%)$ & $4.6(8.2 \%)$ & $3.5(9.1 \%)$ \\
\hline MDRD vs. LM & $4.7(5.6 \%)$ & $9.6(9.9 \%)$ & $1.5(1.9 \%)$ & $1.4(2.6 \%)$ & $2.6(7.7 \%)$ \\
\hline MDRD vs. FAScre & $3.3(3.6 \%)$ & $5.1(4.7 \%)$ & $2.0(2.5 \%)$ & $2.9(5.5 \%)$ & $0.1(0.1 \%)$ \\
\hline CKD-EPIcrecys vs. CKD-EPIcre & $9.9(10.4 \%)$ & $10.1(9.8 \%)$ & $9.8(13.2 \%)$ & $5.4(10.6 \%)$ & $1.9(5.4 \%)$ \\
\hline CKD-EPIcrecys vs. CKD-EPIcys & $6.2(5.6 \%)$ & $6.6(5.7 \%)$ & $4.3(5.3 \%)$ & $1.1(2.0 \%)$ & $0.8(2.3 \%)$ \\
\hline CKD-EPIcrecys vs. LM & $21.4(25.6 \%)$ & $24.0(26.1 \%)$ & $17.6(24.8 \%)$ & $8.3(16.6 \%)$ & $4.8(14.9 \%)$ \\
\hline CKD-EPIcrecys vs. FAScre & $13.4(14.6 \%)$ & $12.6(12.3 \%)$ & $16.2(22.8 \%)$ & $9.4(19.0 \%)$ & $2.0(5.6 \%)$ \\
\hline CKD-EPIcre vs. CKD-EPIcys & $16.1(14.5 \%)$ & $16.8(14.2 \%)$ & $14.6(16.8 \%)$ & $3.5(6.2 \%)$ & $3.4(9.0 \%)$ \\
\hline CKD-EPIcre vs. LM & $11.5(13.7 \%)$ & $15.0(15.8 \%)$ & $8.2(11.0 \%)$ & $3.3(6.3 \%)$ & $2.9(8.9 \%)$ \\
\hline CKD-EPIcre vs. FAScre & $3.5(3.8 \%)$ & $2.2(2.1 \%)$ & $5.6(7.4 \%)$ & $4.6(8.9 \%)$ & $0.4(1.1 \%)$ \\
\hline CKD-EPIcys vs. LM & $27.6(33.0 \%)$ & $30.1(33.5 \%)$ & $22.1(32.2 \%)$ & $7.0(13.9 \%)$ & $6.1(18.7 \%)$ \\
\hline CKD-EPlcys vs. FAScre & $19.7(21.4 \%)$ & $19.4(19.4 \%)$ & $22.3(32.7 \%)$ & $8.0(16.0 \%)$ & $3.0(8.7 \%)$ \\
\hline LM vs. FAScre & $8.0(8.7 \%)$ & $13.5(12.4 \%)$ & $3.5(4.4 \%)$ & $1.2(2.3 \%)$ & $2.1(5.7 \%)$ \\
\hline
\end{tabular}

Relative changes (\%) of the estimated GFR were calculated as ([first value] - [second value]) / [second value])

MDRD Modification of Diet in Renal Disease study equation, CKD-EPIcre Chronic Kidney Disease Epidemiology Collaboration creatinine equation, CKD-EPIcys

Chronic Kidney Disease Epidemiology Collaboration cystatin C equation, CKD-EPIcrecys Chronic Kidney Disease Epidemiology Collaboration creatinine and cystatin

$C$ equation, LM Lund-Malmö equation, FAScre Full Age Spectrum creatinine equation

${ }^{a}$ GFR categories are defined according to the mean value of GFR estimated by the equations being compared (expressed in $\mathrm{ml} / \mathrm{min} / 1.73 \mathrm{~m}^{2}$ ) as follows: $\mathrm{G} 1: \geq 90$, G2: $60<90$, G3a: $45<60$, G3b-G5: $<45$

participants with mean eGFR $<45 \mathrm{ml} / \mathrm{min} / 1.73 \mathrm{~m}^{2}(\mathrm{G} 3 \mathrm{~b}-$ G5), the md ranged from 0.1 (MDRD vs. FAScre) to $6.1 \mathrm{ml} / \mathrm{min} / 1.73 \mathrm{~m}^{2}$ (CKD-EPIcys vs. LM), while among participants with mean eGFR $>90 \mathrm{ml} / \mathrm{min} / 1.73 \mathrm{~m}^{2}$ (G1) the md ranged from 2.2 (CKD-EPIcre vs. FAScre) to $30.1 \mathrm{ml} / \mathrm{min} / 1.73 \mathrm{~m}^{2}$ (CKD-EPIcys vs. LM). Very good levels of agreement between the equations for small values of eGFR were shown also from the Bland-Altman plots that depict the agreement over the whole range of eGFR values (Figs. 3 and 4, and Additional file 1: Figure S1). The greatest variability of the differences between eGFR, estimated as the range between the limits of the agreement, was observed comparing CKD-EPIcys with MDRD (95\% CI: -97.6, 143.5).

\section{Discussion}

This study shows that prevalence estimates of decreased kidney function (eGFR $<60 \mathrm{ml} / \mathrm{min} / 1.73 \mathrm{~m}^{2}$ ) among adults varies considerably depending on the equation used for estimating GFR. Prevalence estimates among of persons aged 18-79 in Germany 2008-2011 (DEGS1) varied from $2.1 \%$ using CKD-EPIcys to $6.9 \%$ using FAScre and the overall number of persons with decreased kidney function ranged accordingly from $2.15 \mathrm{~m}$ (CKDEPIcys) to $6.78 \mathrm{~m}$ (FAScre). From a public health standpoint, the choice of the equation produces a wide range of the estimated number of persons with kidney disease in Germany.

Prevalence estimates of decreased kidney function vary substantially both within and between countries and many potential factors leading to these variations have been discussed [27-29]. Our estimated prevalence is lower than those from the SHIP-1 study and the KORA F4 study, which reported a prevalence of decreased kidney function in Northeast and Southern Germany of $5.9 \%$ and $3.1 \%$ respectively using CKD-EPIcrecys (vs. $2.3 \%$ in DEGS1) [16]. If compared with DEGS1, the median age of participants as well as the prevalence of hypertension and diabetes mellitus in these studies was considerably higher, especially for SHIP-1. Therefore, the observed differences are mostly due to differences in age and in prevalence of risk factors among the study populations. Prevalence estimates in our study were also lower than those from the US population based on the National Health and Nutrition Examination Survey (NHANES), which reported a prevalence estimate of $8 \%$ using MDRD [2]. Some reasons for these differences have been discussed in our previous publication, including heterogeneity in age distribution and ethnic characteristics of the study populations [15].

As the GFR estimating equations include the same demographic variables, such as age and sex, the 

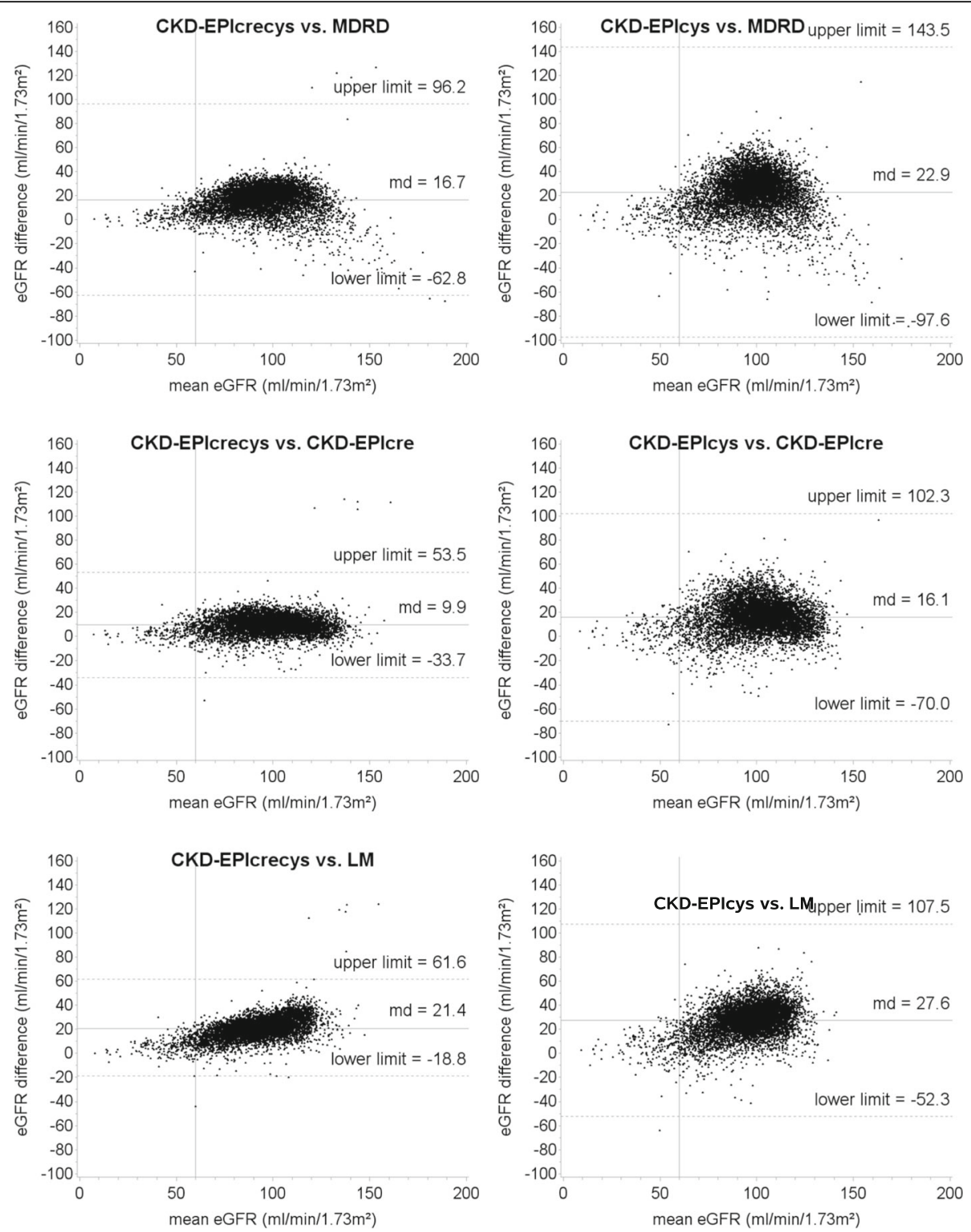

Fig. 3 Bland-Altman plots for comparison between equations that include cystatin C and equations based on creatinine alone among 7001 adults aged 18-79 in Germany 2008-2011 (DEGS1). MDRD: Modification of Diet in Renal Disease study equation; CKD-EPIcre: Chronic Kidney Disease Epidemiology Collaboration creatinine equation; CKD-EPIcys: Chronic Kidney Disease Epidemiology Collaboration cystatin C equation; CKD-EPIcrecys: Chronic Kidney Disease Epidemiology Collaboration creatinine and cystatin C equation; LM: Lund-Malmö equation. Solid, horizontal lines represent the mean difference between the eGFR. Dashed, horizontal lines represent the limit of agreement between the equations. Solid, vertical lines represent the eGFR cut-off value for a decreased kidney function $\left(60 \mathrm{ml} / \mathrm{min} / 1.73 \mathrm{~m}^{2}\right)$

observed differences between prevalence estimates could be mainly due to the fact that some equations use serum cystatin C (CKD-EPIcys, CKD-EPIcrecys), while other equations use serum creatinine alone as biomarker (MDRD, CKD-EPIcre, LM and FAScre). In particular, the prevalence of participants with decreased kidney function estimated by those equations that include serum cystatin $C$ was considerably lower than the prevalence estimated by those equations based on serum creatinine alone. The lowest prevalence was estimated using the equation that includes cystatin $\mathrm{C}$ alone as laboratory parameter (CKD-EPIcys). These results are in line with published findings [30] and may reflect that there are less non-renal factors influencing cystatin $\mathrm{C}$ plasma levels than there are for creatinine plasma levels. Higher prevalence estimates using equations with creatinine alone were found also by the Berlin Initiative Study (BIS) for people aged 70 years and older [17]. In contrast to our study, data based on NHANES showed that equations with creatinine alone yielded lower 

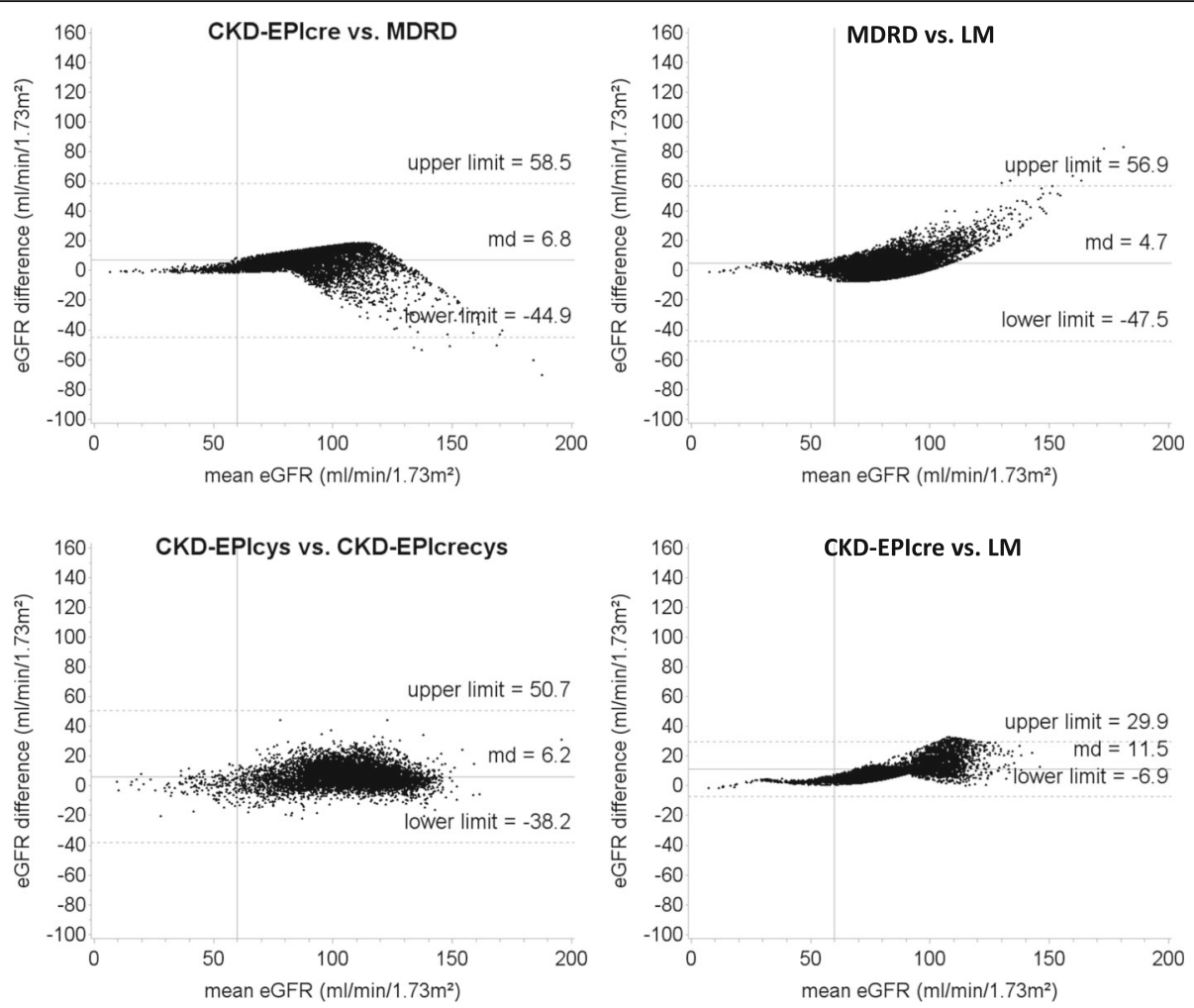

Fig. 4 Bland-Altman plots for comparison between equations both based on cystatin $C$ and comparison between equations both based on creatinine alone among 7001 adults aged 18-79 in Germany 2008-2011 (DEGS1). MDRD: Modification of Diet in Renal Disease study equation; CKDEPIcre: Chronic Kidney Disease Epidemiology Collaboration creatinine equation; CKD-EPIcys: Chronic Kidney Disease Epidemiology Collaboration cystatin C equation; CKD-EPlcrecys: Chronic Kidney Disease Epidemiology Collaboration creatinine and cystatin C equation; LM: Lund-Malmö equation. Solid, horizontal lines represent the mean difference between the eGFR. Dashed, horizontal lines represent the limit of agreement between the equations. Solid, vertical lines represent the eGFR cut-off value of a decreased kidney function $\left(60 \mathrm{ml} / \mathrm{min} / 1.73 \mathrm{~m}^{2}\right)$

prevalence estimates if compared with equations that included cystatin $C$ [31]. Interestingly, in agreement with DEGS1, NHANES reported higher mean values of eGFR for equations with cystatin $C$ if compared with mean eGFR calculated by equations with creatinine alone. Our data point out that the seemingly low level of imprecision of the creatinine based calculations may translate into quite relevant differences when using the equations for epidemiological questions and support the suggestion to use the GFR values estimated by equations with cystatin $\mathrm{C}$ as confirmatory test for people with decreased kidney function as estimated by equations with creatinine only [4].

Given the large difference between the prevalence estimates yielded by the different GFR estimating equations, the choice for the equation for assessing GFR can have a great impact on the assessment of public health implications, e. g. projections of disease burden or medical resources in relation to CKD. Individually, misclassification of patients as having chronic kidney disease can result in unnecessary diagnostic and therapeutic interventions with consequent added costs to the health care system. Actually, the costs of cystatin $\mathrm{C}$ tests vary from about 3 to 20 times those of creatinine tests [32]. It is well known that any estimating equation performs better in those populations which are alike the population in which the equation was developed. For example, the CKDEPIcre equation was validated in a population with a majority of healthy people and therefore this equation provides accurate estimates at higher ranges of eGFR. In contrast, as the MDRD equation was developed in patients with CKD, this equation performs better in populations with lower eGFR. Finally, the BIS2 equation was explicitly designed to accurately estimate GFR in persons aged 70 years or older and should be therefore used in older populations. Therefore, in order to minimize errors in GFR estimations and to reduce the risk of misclassification the equation should be used for which the development population matches best with the population of interest. In our study, great differences between eGFR were calculated by comparing equations that include cystatin $\mathrm{C}$ (CKD-EPIcrecys, CKD-EPIcys) with equations based 
on creatinine alone (MDRD, CKD-EPIcre, LM and FAScre). However, the analysis of the agreement between the equations stratified by the mean values of eGFR shows that the absolute and relative change (percent change) of eGFR was larger among GFR categories G1 and G2 than G3b-G5. In particular, the systematic differences between eGFR among participants classified in the category G3b-G5 can be easily considered clinically not relevant. The Bland-Altman plots showed a similar distribution pattern, with good agreement between the estimating equations for low values of eGFR and increasing systematic differences with increasing eGFR. These results are consistent with those observed in other studies [33, 34] and suggest that the different estimating equations may be used interchangeably among persons with moderately to severely decreased kidney function (eGFR: $<45 \mathrm{ml} /$ $\left.\min / 1.73 \mathrm{~m}^{2}\right)$. Furthermore, high variability of the differences, estimated by the limits of the agreement between the equations, was observed by comparing CKD-EPIcys with equations based on creatinine alone.

This study has some limitations: First, as we did not measure the GFR by a gold standard, we could not determine which equation provides the most valid prevalence estimates of decreased kidney function for the German population. Second, information on place of residence of the study participants was not available and we could not therefore evaluate regional variability in prevalence estimates. Third, as the first wave of the DEGS study was conducted from 2008 to 2011, serum cystatin $\mathrm{C}$ concentration was measured using a not standardized assay which complicates the comparison with studies that used a standardized assay for cystatin C. A further limitation of our and other cross-sectional studies is the lack of a second GFR estimation after 3 months which most likely results in a false positive prevalence of kidney dysfunction.

\section{Conclusions}

Our study illustrates the importance of the choice of the GFR estimating equation from an epidemiological point of view. Prevalence estimates of decreased kidney function in Germany are highly related to the equation used. In particular, the equations that include serum cystatin $\mathrm{C}$ provide lower prevalence estimates if compared with those based on serum creatinine alone. However, the analysis of the systematic differences between the eGFR suggests that the equations could be used interchangeably among persons with pronounced decreased kidney function. Additional longitudinal epidemiological studies are needed to investigate which of the available equations are most useful for prediction of CKD and associated complications at the population level.

\section{Additional file}

Additional file 1: Figure S1. Bland-Altman plots for comparison between Full Age Spectrum creatinine equation (FAScre) and the other equations used to estimate GFR among 7001 adults aged 18-79 in Germany 2008-2011 (DEGS1). MDRD: Modification of Diet in Renal Disease study equation; CKD-EPIcre: Chronic Kidney Disease Epidemiology Collaboration creatinine equation; CKD-EPIcys: Chronic Kidney Disease Epidemiology Collaboration cystatin C equation; CKD-EPIcrecys: Chronic Kidney Disease Epidemiology Collaboration creatinine and cystatin C equation; LM: Lund-Malmö equation; FAScre: Full Age Spectrum creatinine equation. Solid, horizontal lines represent the mean difference between the eGFR. Dashed, horizontal lines represent the limit of agreement between the equations. Solid, vertical lines represent the eGFR cut-off value of a decreased kidney function $\left(60 \mathrm{ml} / \mathrm{min} / 1.73 \mathrm{~m}^{2}\right)$. (PDF $\left.422 \mathrm{~kb}\right)$

\section{Abbreviations}

Cl: Confidence interval; CKD: Chronic Kidney Disease; CKD-EPIcre: Chronic Kidney Disease Epidemiology Collaboration creatinine equation; CKDEPIcrecys: Chronic Kidney Disease Epidemiology Collaboration creatinine and cystatin C equation; CKD-EPIcys: Chronic Kidney Disease Epidemiology Collaboration cystatin C eq.; CV: Coefficient of variation; DEGS: German Health Interview and Examination Survey for Adults; DEGS1: German Health Interview and Examination Survey for Adults, first wave; eGFR: estimated glomerular filtration rate; FAScre: Full Age Spectrum creatinine equation; GFR: Glomerular filtration rate; GNHIES98: German National Health Interview and Examination Survey 1998; Hg: Mercury; IDMS: Isotope Dilution Mass Spectrometry; LM: Lund-Malmö equation; max: Maximum; md: Mean difference; MDRD: Modification of Diet in Renal Disease study equation; min: Minimum; RKI: Robert Koch-Institute; Scr: Serum creatinine; Scys: Serum cystatin C; SD: Standard deviation

\section{Acknowledgements}

The authors thank Angelika Schaffrath Rosario of the Robert Koch-Institute, Berlin, for helpful comments on the statistical analyses of the data.

\section{Availability of data and materials}

Any additional information required by the reader can be obtained from authors upon reasonable request.

\section{Authors' contributions}

PT drafted the manuscript and carried out the statistical analysis, MG and SM made substantial contributions to the analysis and interpretation of the data and revised the manuscript, CSN collected the data, provided intellectual content of critical importance and revised the manuscript, AS was responsible for the design of the study, data analysis and interpretation of the findings and revised the manuscript. All authors read and approved the final manuscript.

\section{Ethics approval and consent to participate}

All participants provided written informed consent prior to the interview and examinations. All procedures performed in DEGS1 were in accordance with the 1964 Helsinki declaration and its later amendments. The study protocol was approved by the Charité-Universitätsmedizin. Berlin ethics committee in September 2008 (No. EA2/047/08).

Consent for publication

Not applicable

Competing interests

The authors declare that they have no competing interests.

\section{Publisher's Note}

Springer Nature remains neutral with regard to jurisdictional claims in published maps and institutional affiliations. 


\section{Author details}

${ }^{1}$ Center of Clinical Epidemiology, c/o Institute of Medical Informatics, Biometry and Epidemiology (IMIBE), University Hospital Essen, Hufelandstr. 55, 45147 Essen, Germany. ${ }^{2}$ Department of Internal Medicine II, Medical Faculty of the Martin-Luther-University Halle-Wittenberg, Ernst-Grube-Str. 40 06120 Halle (Saale), Germany. ${ }^{3}$ Department of Epidemiology and Health Monitoring, Robert Koch-Institute, General-Pape-Str. 62-66, 12101 Berlin, Germany. ${ }^{4}$ Department of Epidemiology, School of Public Health, Boston University, 715 Albany St, Boston, MA 02118, USA.

\section{Received: 8 June 2017 Accepted: 10 November 2017}

\section{Published online: 28 November 2017}

\section{References}

1. Kidney Disease. Improving global outcomes (KDIGO) CKD work group: KDIGO 2012 clinical practice guideline for the evaluation and Management of Chronic Kidney Disease. Kidney. Int Suppl. 2013;3:1-150.

2. Coresh J, Selvin E, Stevens LA, Manzi J, Kusek JW, Eggers P, et al. Prevalence of chronic kidney disease in the United States. JAMA. 2007;298:2038-47.

3. Stevens LA, Coresh J, Greene T, Levey AS. Assessing kidney function measured and estimated glomerular filration rate. N Engl J Med. 2006;354: 2473-83.

4. Inker LA, Schmid CA, Tighiouart H, Eckfeldt JH, Feldman HI, Greene T, et al. Estimating glomerular filtration rate from serum creatinine and cystatin $\mathrm{C}$. N Engl J Med. 2012;367:20-9.

5. Levey AS, Coresh J. Chronic kidney disease. Lancet. 2012;379:165-80.

6. Coresh J, Turin TC, Matsushita K, Sang Y, Ballew SH, Appel L, et al. Decline in estimated glomerular filtration rate and subsequent risk of end-stage renal disease and mortality. JAMA. 2014;311:2518-31.

7. Go AS, Chertow GM, Fan D, McCulloch CE, Hsu CY. Chronic kidney disease and the risks of death, cardiovascular events, and hospitalization. N Engl J Med. 2004;351:1296-305

8. Keith DS, Nichols GA, Gullion CM, Brown JB, Smith DH. Longitudinal followup and outcomes among a population with chronic kidney disease in a large managed care organization. Arch Intern Med. 2004;164:659-63.

9. Eckardt KU, Coresh J, Devuyst O, Johnson RJ, Köttgen A, Levey AS, et al. Evolving importance of kidney disease: from subspecialty to global health burden. Lancet. 2013;382:158-69.

10. Earley A, Miskulin D, Lamb EJ, Levey AS, Uhlig K. Estimating equations for glomerular fitration rate in the era of creatinin standardization: a systematic review. Ann Intern Med. 2012;156:785-95.

11. Levey AS, Stevens LA, Schmid CA, Zhang YL, Castro AF, Feldman HI, et al. A new equation to estimate glomerular filtration rate. Ann Intern Med. 2009; 150:604-12.

12. Nyman U, Grubb A, Larsson A, Hansson LA, Flodin M, Nordin G, et al. The revised Lund-Malmö GFR estimating equation outperforms MDRD and CKDEPI across GFR, age, and BMI intervals in a large Swedish population. Clin Chem Lab Med. 2014:52:815-24.

13. Stevens LA, Coresh J, Feldman HI, Greene T, Lash JP, Nelson RG, et al. Evaluation of the modification of diet in renal disease study equation in a large diverse population. J Am Soc Nephrol. 2007;18:2749-57.

14. Meeusen JW, Rule AD, Voskoboev N, Baumann NA, Lieske JC, Cystatin C. Creatinine-based eGFR equation performance depends on patient characteristics. Clin Chem. 2015;61:1265-72.

15. Girndt M, Trocchi P, Scheidt-Nave C, Markau S, Stang A. The prevalence of renal failure. Dtsch Ärztebl Int. 2016;113:85-91.

16. Aumann N, Baumeister SE, Rettig R, Werner WLA, Döring A, Peters A, et al. Regional variation of chronic kidney disease in Germany: results from two population-based surveys. Kidney Blood Press Res. 2015:40:231-43.

17. Ebert N, Jakob O, Gaedeke J, van der Giet M, Kuhlmann MK, Martus P, et al. Prevalence of reduced kidney function and albuminuria in older adults: the berlin initiative study. Nephrol Dial Transplant. 2017;32:997-1005.

18. Kamtsiuris P, Lange M, Hoffmann R, Schaffrath Rosario A, Dahm S, Kuhnert $R$, et al. First wave of the German health interview and examination survey for adults (DEGS1). Sampling design, response, sample weights and representativeness. Bundesgesundheitsbl. 2013;56:620-30.

19. Kurth BM, Lange $C$, Kamtsiuris $P$, Hölling H. Health monitoring at the Robert-Koch-institute. Status and perspectives. Bundesgesundheitsb Gesundheitsforsch Gesundheitsschutz. 2009:52:557-70.

20. Scheidt-Nave C, Kamtsiuris P, Gößwald A, Hölling H, Lange M, Busch MA et al. German health interview and examination survey for adults (DEGS) - design, objectives and implementation of the first data collection wave. BMC Public Health. 2012;12:730.

21. Heidemann C, Du Y, Schubert I, Rathmann W, Scheidt-Nave C. Prävalenz und zeitliche Entwicklung des bekannten Diabetes mellitus. Ergebnisse der Studie zur Gesundheit Erwachsener in Deutschland (DEGS1). Bundesgesundheitsbl. 2013;56:668-77.

22. Neuhauser H, Thamm M, Eliassen MS. Blutdruck in Deutschland 2008-2011. Ergebnisse der Studie zur Gesundheit Erwachsener in Deutschland (DEGS1). Bundesgesundheitsbl. 2013;56:795-801.

23. Levey AS, Coresh J, Greene T, Marsh J, Stevens LA, Kusek JW, et al. Expressing the modification of diet in renal disease study equation for estimating glomerular filtration rate with standardized serum creatinine values. Clin Chem. 2007:53:766-72.

24. Pottel H, Hoste L, Dubourg L, Ebert N, Schaeffner E, Eriksen BO, et al. An estimated glomerular filtration rate equation for the full age spectrum. Nephrol Dial Transplant. 2016;31:798-806.

25. Bland JM, Altman DG. Statistical methods for assessing agreement between two methods of clinical measurement. Lancet. 1986;1:307-10.

26. Medical Netcare $\mathrm{GmbH}$. Jahresbericht Datenanalyse Dialyse für den Gemeinsamen Bundesausschuss. In: Berichtsjahr; 2013. http://www. medical-netcare.de/qsd.php. Accessed 6 Nov 2014.

27. Glassock RJ, Warnock DG, Delanaye P. The global burden of chronic kidney disease: estimates, variability and pitfalls. Nat Rev Nephrol. 2017;13:104-14.

28. Brück K, Stel VS, Gambaro G, Hallan S, Völzke H, Ärnlöv J, et al. CKD prevalence varies across the European general population. J Am Soc Nephrol. 2016;27:2135-47.

29. McCullough K, Sharma P, Ali T, Khan I, Smith WCS, MacLeod A, et al. Measuring the population burden of chronic kidney disease: a systematic literature review of the estimated prevalence of impaired kidney function. Nephrol Dial Transplant. 2012;27:1812-21.

30. Anderson AH, Yang W, Hsu C, Joffe MM, Leonard MB, Xie D, et al. Estimating GFR among participants in the chronic renal insufficiency cohort (CRIC) study. Am J Kidney Dis. 2012;60:250-61.

31. Grams ME, Juraschek SP, Selvin E, Foster MC, Inker LA, Eckfeld JH, et al. Trends in the prevalence of reduced GFR in the United States: a comparison of creatinine- and cystatin C-based estimates. Am J Kidney Dis. 2013;62:253-60.

32. Shlipak MG, Mattes MD, Peralta CA. Update on cystatin C: incorporation into clinical practice. Am J Kidney Dis. 2013;62:595-603.

33. Delanaye P, Cavalier E, Moranne O, Lutteri L, Krzesinski JM, Bruyere O. Creatinine- or cystatin C-based equations to estimate glomerular filtration in the general population: impact on the epidemiology of chronic kidney disease. BMC Nephrol. 2013:14:57.

34. Glaser N, Deckert A, Phiri S, Rothenbacher D, Neuhann F. Comparison of various equations for estimating GFR in Malawi: how to determine renal function in resource limited setting? PLoS One. 2015; doi: 10.1371/journal. pone.0130453.

\section{Submit your next manuscript to BioMed Central and we will help you at every step:}

- We accept pre-submission inquiries

- Our selector tool helps you to find the most relevant journal

- We provide round the clock customer support

- Convenient online submission

- Thorough peer review

- Inclusion in PubMed and all major indexing services

- Maximum visibility for your research

Submit your manuscript at www.biomedcentral.com/submit
) Biomed Central 